\title{
RECENT HIGH RESOLUTION X-RAY SPECTRA \\ OF THE SUN
}

\author{
J. H. PARKINSON, K. EVANS, and K. A. POUNDS \\ Dept. of Physics, University of Leicester, Leicester, England
}

New results are presented from high resolution Bragg crystal spectrometers flown in late 1970 on two Skylark rockets. The first instrument, launched on 24 November 1970 at 2213 UT from Woomera, South Australia, contained two crystal spectrometers, each with an effective area of $50 \mathrm{~cm}^{2}$ and field collimation to $3^{\prime} \mathrm{FWHM}$. This instrument obtained the X-ray spectrum of the quiet corona in the wavelength range 5-14 $\AA$. The second instrument was launched on 6 December 1970 at 1113 UT from Sardinia, Italy, and contained four crystals of $6 \mathrm{~cm}^{2}$, each collimated to $4^{\prime}$ FWHM. This instrument was pointed at a non-flaring active region near $\mathrm{N} 20 \mathrm{~W} 40$ (McMath region 11060), and obtained an X-ray spectrum between 5 and $23 \AA$. This first use of a collimator to limit the field of view has considerably increased the spectral clarity compared with earlier observations by excluding the contributions of other active regions.

In the active region spectra a number of previously unobserved satellite lines close to the helium-like resonance lines have been observed, and are discussed in detail by Parkinson (1971). The principal lines of OVII, O vill, Nex, MgXI and Si XIII have been used to construct an emission measure-temperature model of the emitting region, following the method outlined in Batstone et al. (1970). The results of the calculations show that the region observed here is significantly cooler than the regions observed by Batstone et al. At $2-3 \times 10^{6} \mathrm{~K}$ both sets of data give similar emission measures of $1.5 \times 10^{48}$ whilst at $4-5 \times 10^{6} \mathrm{~K}$ region 11060 requires an emission measure of $5 \times 10^{46}$ $\mathrm{cm}^{-3}$, approximately an order of magnitude less than for the results of Batstone et al. The relative element abundances can be derived by an iterative method to improve the fit of the model to the line intensities. These show good agreement with the results of Pottasch (1967) and Beigman and Vainshtein (1971), confirming that the heavy metal elements become relatively more abundant in the corona than in the photosphere. In the NeIX $1 s^{2}-1 s n p$ series, lines are observed for $2 \leqslant n \leqslant 6$. With the exception of the $n=3$ line, the lines are of the expected strengths relative to the $n=2$ line, on the basis of the oscillator strengths of Dalgarno and Parkinson (1967). Several Fexvir lines were observed and their intensities are broadly in agreement with those of Evans and Pounds (1968).

The best fit to the quiet corona data gives a temperature of $2.0 \times 10^{6} \mathrm{~K}$ with an emission measure of $4 \times 10^{45} \mathrm{~cm}^{-3}$ arc $\mathrm{min}^{-2}$ at the centre of the Sun.

\section{References}

Batstone, R. M., Evans, K., Parkinson, J. H., and Pounds, K. A.: 1970, Solar Phys. 13, 389.

Beigman, I. L. and Vainshtein, L. A. : 1971, Soviet Astron. 14, 825.

Dalgarno, A. and Parkinson, E. M.: 1967, Proc. Phys. Soc. A 301, 253. 
Evans, K. and Pounds, K. A.: 1968, Astrophys. J. 152, 319.

Parkinson, J. H.: 1971, Nature Phys. Sci. 233, 44.

Pottasch, S. R.: 1967, Bull. Astron. Inst. Neth. 19, 113.

\section{DISCUSSION}

T. den Boggende: In earlier crystal spectra of the Sun there was always a considerable enhancement of the 'continuum' around the strong lines at 15.01 and $15.4 \AA$. In your spectra this enhancement is less but still present. Is this unresolved lines or is there another source? Where is the continuum level in this wavelength region?

J. H. Parkinson: I think the apparent enhancement in the continuum between 15.4 and $16.0 \AA$ is due to several lines, probably from transitions in FexviII.

A. H. Gabriel: I was interested in your comment that no quartet satellite lines were observed. My theory predicts that no such lines should result from dielectronic recombination for elements up to above silicon. If any are seen then they must be due to inner-shell excitation.

G. A. Doschek: NRL spectra show a weak but definite emission feature on the long-wavelength side of the SixIII forbidden line during flares. Whether this feature is due to transitions from ${ }^{4 P}$ states in lithium like ions is not yet definitely established.

J. H. Parkinson: Following the remarks of Gabriel and Doschek I can confirm that in the active region spectra no quartet satellite lines are observed in oxygen, neon or magnesium.

A. B. C. Walker: Do you see any Fexvin lines? There is an FexvII line which is coincident with the O vir Lyman- $\beta$ line and makes it difficult to obtain a reliable Lyman- $\beta /$ Lyman- $\alpha$ ratio for oxygen when the Sun is active.

J. H. Parkinson: In the active region spectra the contribution of Fe XvIII to the $16.01 \AA$ blend with $\mathrm{OVII}$ is expected to be less than $5 \%$ for temperatures below $4 \times 10^{6} \mathrm{~K}$.

A. B. C. Walker: What in fact was the OviI Lyman- $\beta /$ Lyman- $\alpha$ ratio?

$J$. H. Parkinson: The O viı Lyman- $\beta /$ Lyman- $\alpha$ ratio from these observation is 0.16 . 